\title{
ARTICLE
}

\section{Vacuum Rabi splitting in a plasmonic cavity at the single quantum emitter limit}

Kotni Santhosh ${ }^{1, \star, \dagger}$, Ora Bitton ${ }^{2, \star}$, Lev Chuntonov ${ }^{3, \star} \&$ Gilad Haran ${ }^{1}$

The strong interaction of individual quantum emitters with resonant cavities is of fundamental interest for understanding light-matter interactions. Plasmonic cavities hold the promise of attaining the strong coupling regime even under ambient conditions and within subdiffraction volumes. Recent experiments revealed strong coupling between individual plasmonic structures and multiple organic molecules; however, strong coupling at the limit of a single quantum emitter has not been reported so far. Here we demonstrate vacuum Rabi splitting, a manifestation of strong coupling, using silver bowtie plasmonic cavities loaded with semiconductor quantum dots (QDs). A transparency dip is observed in the scattering spectra of individual bowties with one to a few QDs, which are directly counted in their gaps. A coupling rate as high as $120 \mathrm{meV}$ is registered even with a single QD, placing the bowtie-QD constructs close to the strong coupling regime. These observations are verified by polarization-dependent experiments and validated by electromagnetic calculations.

\footnotetext{
${ }^{1}$ Department of Chemical Physics, Weizmann Institute of Science, POB 26, Rehovot 76100, Israel. ${ }^{2}$ Department of Chemical Research Support, Weizmann Institute of Science, Rehovot 76100, Israel. ${ }^{3}$ Schulich Faculty of Chemistry, Technion-Israel Institute of Technology, Haifa 32000, Israel. * These authors contributed equally to this work. †Present address: Hindustan Petroleum Green R\&D Center, HPCL, Bangalore 560067, India. Correspondence and requests for materials should be addressed to G.H. (email: gilad.haran@weizmann.ac.il).
} 
T he interaction of emitters with an optical cavity falls within the realm of cavity quantum electrodynamics ${ }^{1}$. Approaching the limit of strong coupling between individual quantum emitters and resonant cavities is important for multiple advanced optical applications, such as quantum information processing 2,3 and quantum communication ${ }^{4,5}$. Strong coupling can be observed through the phenomenon of vacuum Rabi splitting in the optical spectra of the joint systems, as well as in the appearance of non-classical photon correlations. The strength of the interaction depends on the ratio of the quality factor of the cavity to the mode volume, $Q / V$. In optical cavities made of, for example, photonic crystals ${ }^{6}$ or micropillars ${ }^{7}$, the fundamental laws of diffraction set a strict limitation on the cavity size, which cannot be smaller than half the wavelength of the interacting photon. This in turn limits how small $V$ can be and mandates a very high $Q$, to attain the strong coupling regime. Obtaining such a high-quality factor requires demanding experimental conditions such as cryogenic temperatures and ultra-narrow frequency light sources.

Cavities made of materials that can sustain surface plasmon (SP) excitations can beat the diffraction limit by focusing intense electromagnetic fields into volumes much smaller than the wavelength of light ${ }^{8}$. Such cavities should simplify considerably the experimental conditions required for strong light-matter interactions and may allow quantum optical experiments to be conducted under ambient conditions.

Noble metals are most often used for generating structures sustaining SPs at visible wavelengths. Although the SP relaxation times in such structures are ultrafast, severely limiting $Q$, their mode volume is drastically reduced compared with photonic cavities. In recent years, there has been much interest in studies of coupling between SPs, either propagating or localized, and quantum emitters such as molecules?. These experiments involved multiple molecules and often multiple or extended plasmonic structures as well. In recent times, strong coupling has also been probed at the level of a single plasmonic device. Thus, Halas and colleagues ${ }^{10}$ used gold dimers and molecular J-aggregates to observe a Rabi splitting of $\sim 230 \mathrm{meV}$. Shegai and colleagues $^{11}$ observed strong coupling using either a silver nanorod or a silver nanoprism ${ }^{12}$, in both cases employing J-aggregates as the quantum emitters. In all of these experiments, the interaction involved hundreds of quantum emitters or more. However, for quantum information operations one needs to approach the limit of a single quantum emitter coupled to the cavity.

In this study, we show that one can indeed observe strong coupling in the limit of a single quantum emitter. In particular, we use silver bowtie plasmonic cavities and couple them to semiconductor quantum dots (QDs). Scattering spectra registered from individual plasmonic cavities containing one to a few QDs show vacuum Rabi splitting, indicating that the strong coupling regime is approached in these systems. Polarization-dependent experiments verify that the observed Rabi splitting is due to the coupling of the longitudinal plasmon resonance of the bowties with the QDs.

\section{Results}

Construction of plasmonic cavities with QDs. Inspired by recent computational studies, suggesting that strong coupling with individual emitters can be achieved in the gaps of plasmonic structures $^{13}$, we turned to silver bowties, which can be fabricated with a small gap ${ }^{14}$. Silver is preferred as a material for plasmonic devices due to its relatively low SP damping and hence the high achievable quality factors of such devices compared with other metals ${ }^{15}$. By varying the structural parameters of the bowties, including side length and gap size, the localized SP excitations of bowties manufactured by electron-beam lithography can be tuned to resonance with the quantum emitter of choice, while maintaining gaps of the order of $\sim 20 \mathrm{~nm}$. To achieve strong coupling, the quantum emitter should possess a large oscillator strength and a narrow linewidth. QDs have several advantages over organic emitters in relation to the aforementioned characteristics $^{16}$ and (most importantly) are also significantly more photostable. They can also be observed by electron microscopy and therefore can be directly counted. Commercially available CdSe/ZnS QDs were employed in the present study. Optical spectroscopy and electron microscopy studies showed that the sample is mildly heterogeneous and the diameter of the QD varies between 6 and $8 \mathrm{~nm}$.

To position QDs within the gaps of bowties, we made use of interfacial capillary forces ${ }^{17}$ to drive the QDs into lithographically patterned holes in the bowtie gaps. This simple method avoids additional steps such as chemical modification of the QD/nanostructure surfaces. The process, described in Fig. 1 and in the Methods section, led to the trapping in bowtie gaps of a number of QDs that varied from one to several, as could be ascertained by scanning electron microscopy.

Spectroscopy demonstrates Rabi splitting. Dark-field microspectroscopy was used to characterize the plasmonic behaviour of every single bowtie by measuring its scattering spectrum. As shown in Supplementary Fig. 1, empty bowties possess two modes, a transverse mode at $\approx 2.05 \mathrm{eV}$ and a longitudinal mode at $\approx 1.9 \mathrm{eV}$, the latter being due to dipolar coupling of the two parts of each bowtie. The absorption and emission spectra of the QDs are shown in Supplementary Fig. 2 and demonstrate that the optical transitions of the QDs are in resonance with the plasmon excitations of the bowties. (See also Supplementary Note 1 for further discussion on the role of the absorption and emission spectra in coupling to the plasmonic cavity.)

Figure 2a shows bowties with QDs in their gaps whose scattering spectra present transparency dips indicative of Rabi

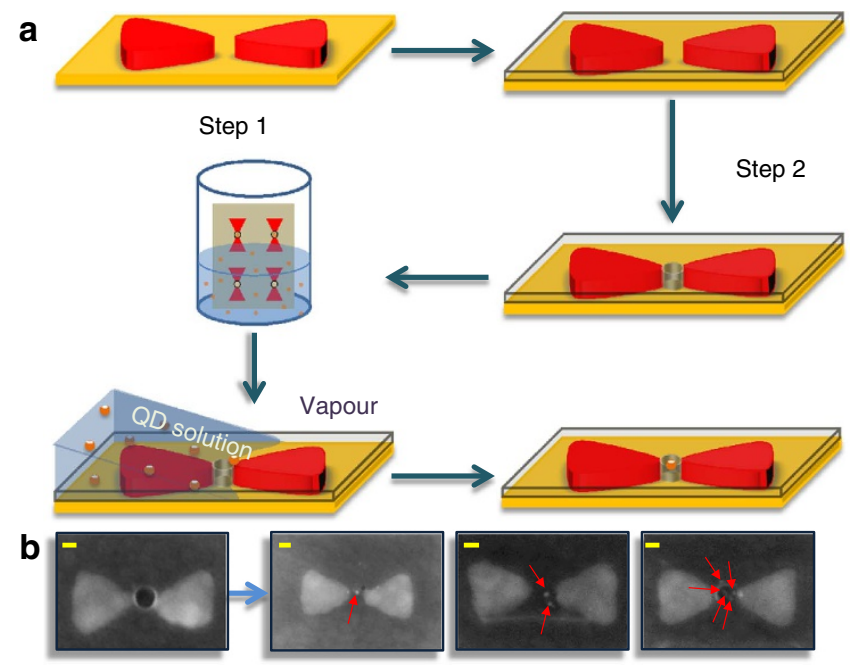

Figure 1 | Construction of bowties with quantum dots in their gaps.

(a) Schematic illustration of the two-step lithography process for making holes at the centre of bowtie structures and the interfacial capillary force assisted method for driving QDs into the holes. (b) Scanning electron microscope images of bowties with one, two and multiple QDs in their gaps (from left to right). The positions of the QDs are marked by red arrows. Scale bars, $20 \mathrm{~nm}$ (yellow). 


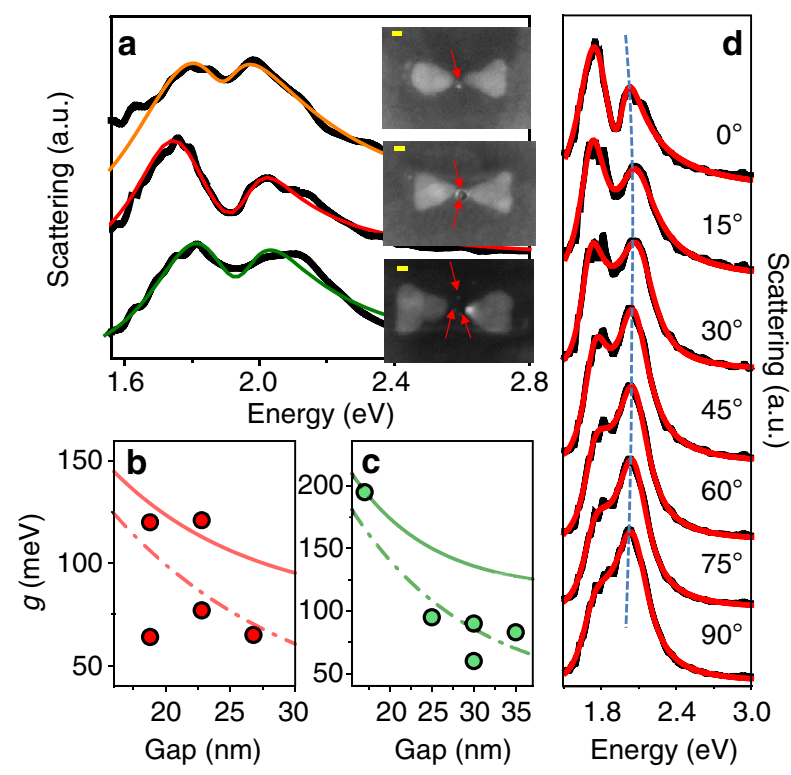

Figure 2 | Strong coupling of plasmons and quantum emitters.

(a) Scattering spectra of bowties with (from top to bottom) one, two and three QDs in the gap, respectively. All spectra show a transparency dip due to Rabi splitting. The black lines are experimental data and the coloured lines are fits to the coupled oscillator model. Insets show the scanning electron microscope images of the bowties. The positions of the QDs are marked by red arrows. Scale bars, $20 \mathrm{~nm}$ (yellow). (b,c) Coupling rates as a function of gap size for bowties with one QD ((b) red symbols) and two QDs ((c) green symbols). The errors in the coupling rate values, obtained from the fitted functions, are estimated to be $2-5 \mathrm{meV}$. The continuous lines represent the numerically calculated coupling rates at two configurations along the centre line of the bowtie: with the QDs almost touching one of the prisms (continuous lines) or with the QDs at the centre of the bowtie (dashed-dotted lines). In the experiments, the QDs may be positioned away from the centre line so that their coupling rates are lower than the calculated lines. (d) Polarization series of the middle bowtie structure in a. As the polarization of the excitation light is rotated from the direction parallel to the bowtie long axis to perpendicular to it, the transparency dip in the spectrum gradually vanishes, indicating that it indeed originates in the coupling of the QD exciton with the longitudinal plasmon resonance of the bowtie.

splitting. Overall, we collected scattering spectra from 21 QDcontaining bowties, out of which 14 spectra showed a definite signature of splitting. Thus, the top bowtie in Fig. 2a, with a single QD and a particularly small gap $(\sim 19 \mathrm{~nm})$, presents a clear Rabi splitting feature. Similarly, Rabi splitting is observed in the spectra of the two other bowties shown in Fig. 2a, with two and three QDs and gaps of 17 and $30 \mathrm{~nm}$, respectively. The spectra in this figure, as well as other spectra showing Rabi splitting, were fitted using the coupled oscillator model ${ }^{18}$ (see Methods). This model represents the plasmon mode and the exciton of the quantum emitter as coupled harmonic oscillators exchanging energy reversibly. The coupling rate characterizing the interaction is given by $g=\boldsymbol{\mu} \cdot \mathbf{E}$, where $\boldsymbol{\mu}$ is the transition dipole moment of the exciton and $\mathbf{E}$ is the electric field of the plasmon. This coupling leads to the generation of upper $\left(\Omega_{+}\right)$and lower $\left(\Omega_{-}\right)$ plasmon-exciton hybrid states, with a transparency dip in between ${ }^{13,19}$. The Rabi splitting values, $\Omega_{+}-\Omega_{-}$, can be obtained directly from the fitted curves, and for the three examples in Fig. 2a they are 176, 288 and $224 \mathrm{meV}$, respectively. These values are comparable to recently reported values of Rabi splittings of J-aggregates with individual plasmonic structures $^{10,12}$, although hundreds of quantum emitters were involved in the latter. Not all bowties loaded with a single QD showed a clear transparency dip in the scattering spectrum. Instead, Rabi splitting manifested itself in a significant spectral broadening as compared with that of an empty bowtie (Supplementary Fig. 3), indicating coupling between plasmons and excitons. Splitting was readily observed in bowties with several QDs; see Supplementary Fig. 4 for additional spectra.

Coupling rates obtained from the fits of data sets with one and two QDs are plotted as a function of gap size in Fig. 2b,c. The figures also contain theoretical estimates of the coupling rates, based on the numerical calculations described below. The coupling rate depends strongly on the gap size of each bowtie. For instance, for two QDs the coupling rate is more than doubled when the gap size changes from 25 to $17 \mathrm{~nm}$. In general, the coupling increases with the number of quantum emitters $(N)$ $\left(g \approx \sqrt{N} g_{1}, g_{1}\right.$ being the coupling rate for one quantum emitter $\left.{ }^{1}\right)$, although in our experiments this dependence is partially masked by the dependence on gap size and QD position variations. Although the values of the coupling rates obtained from the fits are smaller than the values of the plasmon widths (which are $\sim 400 \mathrm{meV}$ ), significant splittings are observed, indicating that the strong coupling regime has indeed been attained, or is close ${ }^{9}$. Supplementary Fig. 5 shows histograms of the values of all parameters obtained from the coupled oscillator fits to measured spectra.

To obtain an unequivocal proof that the splitting in the spectra is due to strong (or close-to-strong) coupling of the bowtie longitudinal plasmon mode and the quantum emitter's exciton, we resorted to polarization-dependent experiments in which the polarization of the excitation source was rotated. Figure $2 \mathrm{~d}$ shows an example of a polarization series measured on the bowtie with two QDs from Fig. 2a. When the excitation is polarized along the longitudinal direction of the bowtie (that is, along its long axis), the spectrum shows two peaks due to Rabi splitting. As the polarization is rotated to the transverse direction, the double-peaked spectrum is gradually replaced with the single-peaked spectrum of the transverse mode. Similar results with other bowties are shown in Supplementary Fig. 6 . These results clearly indicate that the transparency dips in the scattering spectra are due to a genuine coupling of the plasmon and QD excitations.

\section{Discussion}

The experimental results described above show that the positioning of one or a few QDs within a silver bowtie can bring this plasmonic cavity very close to the strong coupling regime. To support the experimental results and shed further light on them, we performed electromagnetic calculations, using the boundary element method ${ }^{20}$. The simulations revealed some interesting facts. The top spectrum in Fig. 3a is the calculated scattering spectrum of an empty bowtie. The spectrum for a bowtie with a single QD at the centre of the gap is shown below, demonstrating a small change compared with the empty bowtie. However, when the QD is positioned closer to one of the prisms constituting the bowtie, or the number of QDs in the hotspot is increased from one to two, a more significant transparency dip appears. The Rabi splitting is observed in both calculated scattering and extinction spectra (the latter not shown). Importantly, no such splitting is found when the simulation is repeated with a single metallic prism rather than a bowtie structure.

The simulation allows us to directly calculate the coupling rate for a quantum emitter with an oscillator strength similar to a QD $(\sim 0.6)^{21-23}$. The spatial distribution of the coupling rate across the gap is shown in Fig. 3b, clearly indicating that the 

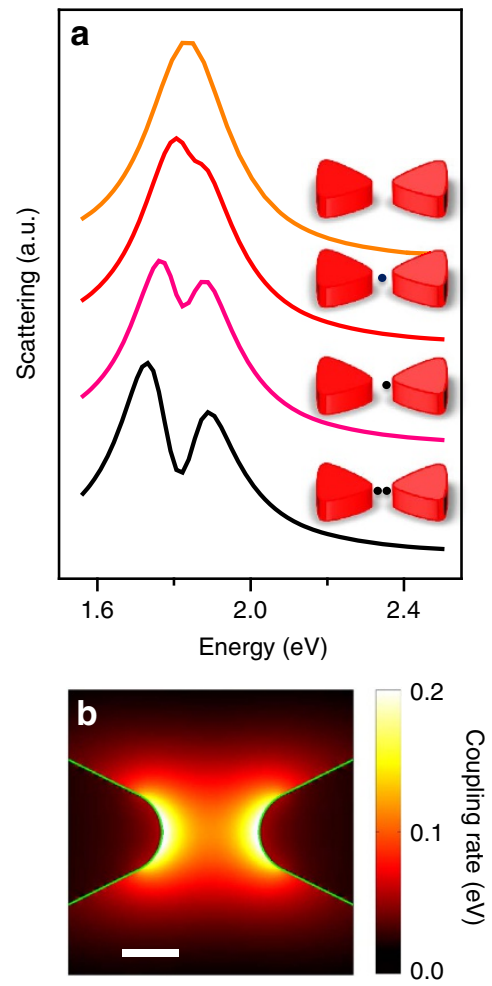

Figure 3 | Electromagnetic simulations of strongly coupled plasmonic structures. (a) Simulated scattering spectra of bowties with one and two QDs organized as indicated in the inset structures. (b) Distribution of the coupling rate (in $\mathrm{eV}$ ) of a quantum emitter with an oscillator strength of 0.6 in a bowtie structure. The white bar represents $10 \mathrm{~nm}$.

electromagnetic field in the hotspot is not uniform and is concentrated at the edges rather than at the centre. Indeed, in many of the bowties exhibiting strong coupling (Fig. 2 and Supplementary Figs 3 and 4) the QDs are close to one of the edges, in agreement with the simulation results. We estimate from the simulation that at the centre of a bowtie structure $g$ is $\sim 100 \mathrm{meV}$, whereas $g$ is as high as $200 \mathrm{meV}$ very close to one of the prisms.

In conclusion, we have successfully integrated lithographically fabricated silver bowties with one to a few QDs that reside exactly within the plasmonic cavity. The very small plasmon mode volumes of the bowties allowed us to demonstrate vacuum Rabi splitting in the limit of an individual quantum emitter. A key advantage of using QDs is that we can directly count them under the electron microscope and verify the relation between coupling and the number of quantum emitters within each cavity. An important question to ask is: what value of the coupling may deem it 'strong'? An often-used rule of thumb for the strong coupling regime is $2 g>\left(\gamma_{0}+\gamma_{\mathrm{pl}}\right) / 2$, where $\gamma_{0}$ and $\gamma_{\mathrm{pl}}$ are the $\mathrm{QD}$ and plasmonic cavity linewidths, respectively ${ }^{9,24}$. The maximal coupling rates obtained in this work for two and three QDs ( 200 meV; Fig. 2b,c and Supplementary Fig. 5d) fulfil the above criterion, whereas the maximum value obtained for a single QD $(120 \mathrm{meV})$ is somewhat smaller. This shows that the constructs of QDs with plasmonic cavities studied here are definitely close to the strong coupling regime. In future experiments we plan to significantly increase the coupling between individual QDs and bowties. To this end, we will reduce the mode volume of the bowties, which can be achieved by decreasing their gap size and improving control over their shape in the fabrication process. Further improvement in QD positioning methodology will allow us to locate a single
QD reproducibly at the position of the highest field within a bowtie cavity. Such improvements will enable systematic variation of experimental parameters and therefore provide additional proofs for strong coupling. Importantly, our results also pave the way to more sophisticated quantum nonlinear optics experiments ${ }^{25}$. In particular, by probing time-resolved photoluminescence of the coupled QD-bowtie system we hope to directly demonstrate quantum correlations ${ }^{2,26}$.

\section{Methods}

Materials. Glass/ITO $(10 \times 10 \times 1.1 \mathrm{~mm})$ substrates were purchased from Xin Yan Technology Ltd., China. The ITO layer is $180 \mathrm{~nm}$ thick and is characterized by $83 \%$ transparency to visible light and a sheet resistance of $10 \Omega^{-2}$. Poly(methyl methacrylate) $950 \mathrm{~K} \mathrm{~A} 2$ (PMMA) electron beam resist, used for the fabrication of bowties, was procured from MicroChem. Chromium (used as an adhesive layer) and silver were obtained from Kurt J. Lesker. Water-soluble mercapto-undecanoic acid capped CdSe/ZnS core/shell nanocrystals (QDs) were acquired from MK Impex Corp.

Electron beam lithography and fabrication of silver bowties. Before fabrication, glass/ITO substrates were cleaned with acetone followed by isopropanol for $3 \mathrm{~min}$ in each step. The cleaned glasses were dried using a nitrogen flow and then PMMA was spin-coated at a speed of 6,000 r.p.m. for $50 \mathrm{~s}$ to achieve a thickness of $60 \mathrm{~nm}$, followed by baking at $180^{\circ} \mathrm{C}$ for $90 \mathrm{~s}$. The PMMA-coated glasses were loaded into a Raith E_line Plus electron beam lithography system and the PMMA was exposed to define the shape of bowties. The accelerating voltage used for the exposure was $30 \mathrm{kV}$ and the beam current was $30 \mathrm{pA}$. The design consisted of matrices of bowties, with each matrix hosting 64 bowties. Each bowtie was separated by $10 \mu \mathrm{m}$ from its neighbouring partner to guarantee no interaction. To remove the exposed PMMA, the substrates were developed using 1:3 methyl isobutyl ketone:isopropyl alcohol for $30 \mathrm{~s}$, followed by immersion in a stopper (isopropyl alcohol) for $30 \mathrm{~s}$ and drying with a nitrogen flow.

An electron beam evaporator (Odem) was used for the metal deposition on the patterned substrates. Initially, chromium was evaporated to deposit a $2-\mathrm{nm}$ adhesion layer, followed by silver. Different prism lengths from 75 to $200 \mathrm{~nm}$ and thicknesses from 20 to $35 \mathrm{~nm}$ were examined and finally the dimensions were fixed to a length of $\approx 85 \mathrm{~nm}$ and a thickness of $\approx 30 \mathrm{~nm}$. These bowties showed a plasmon resonance that overlapped the QD exciton frequency. After metallization, a lift-off step was performed in acetone for $5 \mathrm{~min}$ to obtain silver bowties on the glass/ITO substrate.

A second stage of E-beam lithography was performed, to expose holes in the gap regions of the bowties. A 60-nm layer of PMMA was spin-coated on the substrate and then the gap regions of the bowties were exposed to create 10-30 nm holes, into which the QDs were driven. (See below and in Fig. 1 of the main text.) To reach an overlay accuracy as high as a few nanometres in the second exposure, we made use of alignment marks that were fabricated in the first E-beam exposure step.

Incorporation of QDs into bowtie structures. To incorporate QDs into the bowtie gaps we adopted a method originally developed by Alivisatos and colleagues $^{17}$. Substrates with bowties and holes in the PMMA layer prepared as described above were placed vertically in an aqueous solution of QDs. Controlled solvent evaporation was then used to exert a capillary force along the receding line of contact of the QD solution and drive QDs successfully into the holes. By varying the QD concentration in solution (from 10 to $100 \mathrm{nM}$ ), bowties with one to a few QDs in the gap were successfully obtained (Fig. 1). Although this method is limited in controlling the exact position of the QDs within the gap, it was found to be facile and relatively reproducible.

Bulk absorption and emission measurements of QDs. Steady-state absorption and emission spectral measurements (Supplementary Fig. 2) were carried out on a Cary-100 ultraviolet-visible spectrophotometer (Varian) and a Fluorolog-3 spectrofluorimeter (Horiba Jobin Yvon), respectively. Extinction coefficients of the QDs were estimated by employing the method developed by Peng and colleagues ${ }^{23}$

Dark-field microspectroscopy. Our dark-field microspectrometer is described schematically in Supplementary Fig. 8. Briefly, scattering spectra of single bowties, either with or without QDs, were measured with an inverted microscope equipped with a dark-field condenser, a $75 \mathrm{~W}$ Xenon lamp (Olympus) and a $\times 100$ oil-immersion objective with a numerical aperture of 0.6. A SpectraPro-150 spectrograph with a $1,200 \mathrm{~g} \mathrm{~mm}^{-1}$ grating (Acton) and a Newton spectroscopy charge-coupled device camera (Andor Technology) were used to disperse scattered photons and register spectra. Raw spectra were smoothed using a Fourier low-pass filter. Before the spectral measurements, the camera was calibrated in reference to the spectrum of fluorescent dyes. Polarization of the excitation light was controlled by a combination of a polarizer and a selecting sector, such that essentially only $s$-polarized light reached the sample. 
Data fitting to the coupled oscillator model. Scattering spectra were fitted to the following equation, which represents the scattering of two coupled harmonic oscillators at frequency $\omega^{18}$ :

$$
\sigma_{\mathrm{sca}}(\omega)=A \omega^{4}\left|\frac{\left(\omega^{2}-\omega_{0}^{2}+i \omega \gamma_{0}\right)}{\left(\omega^{2}-\omega_{0}^{2}+i \omega \gamma_{0}\right)\left(\omega^{2}-\omega_{\mathrm{pl}}^{2}+i \omega \gamma_{\mathrm{pl}}\right)-\omega^{2} g^{2}}\right| .
$$

In this expression $\omega_{\mathrm{pl}}$ and $\omega_{0}$ are the plasmon and QD resonance frequencies, respectively, whereas $\gamma_{\mathrm{pl}}$ and $\gamma_{0}$ are the corresponding linewidths. $g$ is the coupling rate and $A$ is a scaling parameter. Parameters obtained from fits to all our data sets are shown in Supplementary Fig. 5. We fixed the value of $\gamma_{0}$ to $130 \mathrm{meV}$, which is the measured linewidth of the fluorescence spectra of individual QDs

(Supplementary Fig. 2). In the case of some of the data sets with a single QD in the gap, where broadening of the spectrum was observed, although no clear splitting, we also fixed the value of $\gamma_{\mathrm{pl}}$ to $385 \mathrm{meV}$, which is the average value of this parameter obtained from multiple spectra of empty bowties.

Electromagnetic simulations. Numerical simulations of the silver bowties were performed using the boundary-element method as implemented in MNPBEM-a toolbox developed by Hohenester and Trügler ${ }^{20}$. A numerical model of a bowtie was constructed from two prisms with equilateral triangular bases of $80 \mathrm{~nm}$ side length, $30 \mathrm{~nm}$ heights, radii of curvature at the vertices of $7 \mathrm{~nm}$ and a gap size of $17 \mathrm{~nm}$. The complex refractive index of silver from Johnson and Christy ${ }^{27}$ was used and a refractive index of 1.33 was assumed for the ambient medium. The bowtie was excited by a plane wave at normal incidence and polarized along the longitudinal direction and the plasmon scattering spectra were calculated at the far field. From the spectrum of an unloaded cavity, we calculated $\gamma_{\mathrm{pl}}=0.25 \mathrm{eV}$, which is smaller than the typical experimental linewidth. However, the cavity quality factor $Q=\omega_{\mathrm{pl}} / \gamma_{\mathrm{pl}}=7.3$ was only slightly higher than the measured quality factor. Simulations of scattering spectra for a bowtie loaded with QDs were performed with dots modelled as spheres of $8 \mathrm{~nm}$ located at various positions within the gap. The complex dielectric function of these dots was approximated by a Lorentz model with a high-frequency dielectric constant $\epsilon_{\infty}=6.1, \omega_{0}=1.8 \mathrm{eV}, \gamma_{0}=0.08 \mathrm{eV}$ and an oscillator strength $f=0.6$ (refs 21-23). As the computed plasmon linewidth of the idealized bowtie structure was consistently smaller than the measured linewidth of a real bowtie, we scaled the linewidth of the QD accordingly.

The position-dependent coupling rate of a QD strongly coupled to the bowtie $g(r)$ was evaluated using $\mathbf{E}(\mathbf{r})$, the electric field numerically calculated at the plasmon resonance energy $\omega_{\mathrm{pl}}=1.83 \mathrm{eV}$. To this end, the mode volume of the antenna was calculated using the approach of Koenderink ${ }^{28}$ and was used to normalize the energy density within the cavity, assuming it is occupied with a single photon of energy $1.83 \mathrm{eV}$. A map of $g(r)$ plotted on a horizontal plane that intersects the bowtie at its half-height is shown in Fig. 3b. Supplementary Fig. 7 shows a similar calculation for a single prism (panel a), and compares the coupling rate of a bowtie and a single prism (panel b).

Data availability. All relevant data are available from the authors on request.

\section{References}

1. Haroche, S. \& Raimond, J. M. Exploring the Quantum: Atoms, Cavities and Photons 1st edn (Oxford Univ. Press, 2006).

2. Hennessy, K. et al. Quantum nature of a strongly coupled single quantum dot-cavity system. Nature 445, 896-899 (2007).

3. Monroe, C. Quantum information processing with atoms and photons. Nature 416, 238-246 (2002).

4. Lo, H. K. \& Chau, H. F. Unconditional security of quantum key distribution over arbitrarily long distances. Science 283, 2050-2056 (1999).

5. Kimble, H. J. The quantum internet. Nature 453, 1023-1030 (2008).

6. Yoshie, T. et al. Vacuum Rabi splitting with a single quantum dot in a photonic crystal nanocavity. Nature 432, 200-203 (2004).

7. Reithmaier, J. P. et al. Strong coupling in a single quantum dot-semiconductor microcavity system. Nature 432, 197-200 (2004).

8. Brongersma, M. L. \& Shalaev, V. M. Applied physics. The case for plasmonics. Science 328, 440-441 (2010).

9. Torma, P. \& Barnes, W. L. Strong coupling between surface plasmon polaritons and emitters: a review. Rep. Prog. Phys. 78, 34 (2015).

10. Schlather, A. E., Large, N., Urban, A. S., Nordlander, P. \& Halas, N. J. Near-field mediated plexcitonic coupling and giant Rabi splitting in individual metallic dimers. Nano Lett. 13, 3281-3286 (2013).

11. Zengin, G. et al. Approaching the strong coupling limit in single plasmonic nanorods interacting with J-aggregates. Sci. Rep. 3, 3074 (2013).
12. Zengin, G. et al. Realizing strong light-matter interactions between singlenanoparticle plasmons and molecular excitons at ambient conditions. Phys. Rev. Lett. 114, 157401 (2015).

13. Savasta, S. et al. Nanopolaritons: vacuum Rabi splitting with a single quantum dot in the center of a dimer nanoantenna. ACS Nano 4, 6369-6376 (2010).

14. Schuck, P. J., Fromm, D. P., Sundaramurthy, A., Kino, G. S. \& Moerner, W. E Improving the mismatch between light and nanoscale objects with gold bowtie nanoantennas. Phys. Rev. Lett. 94, 017402 (2005).

15. Ru, E. C. L. \& Etchegoin, P. G. Principles of Surface Enhanced Raman Spectroscopy (Elsevier, 2009).

16. Leistikow, M. D., Johansen, J., Kettelarij, A. J., Lodahl, P. \& Vos, W. L. Size-dependent oscillator strength and quantum efficiency of CdSe quantum dots controlled via the local density of states. Phys. Rev. B 79, 045301 (2009).

17. Cui, Y. et al. Integration of colloidal nanocrystals into lithographically patterned devices. Nano Lett. 4, 1093-1098 (2004).

18. Wu, X., Gray, S. K. \& Pelton, M. Quantum-dot-induced transparency in a nanoscale plasmonic resonator. Opt. Express 18, 23633-23645 (2010).

19. Rudin, S. \& Reinecke, T. L. Oscillator model for vacuum Rabi splitting in microcavities. Phys. Rev. B 59, 10227-10233 (1999).

20. Hohenester, U. \& Trügler, A. MNPBEM-A Matlab toolbox for the simulation of plasmonic nanoparticles. Comput. Phys. Commun. 183, 370-381 (2012).

21. Alivisatos, A. P., Harris, T. D., Carroll, P. J., Steigerwald, M. L. \& Brus, L. E. Electron-vibration coupling in semiconductor clusters by resonance Raman spectroscopy electron-vibration coupling in semiconductor clusters studied by resonance Raman-spectroscopy. J. Chem. Phys. 90, 3463-3468 (1989).

22. Leistikow, M. D., Johansen, J., Kettelarij, A. J., Lodahl, P. \& Vos, W. L. Size-dependent oscillator strength and quantum efficiency of CdSe quantum dots controlled via the local density of states. Phys. Rev. B 79, 9 (2009).

23. Yu, W. W., Qu, L., Guo, W. \& Peng, X. Experimental determination of the extinction coefficient of CdTe, CdSe, and CdS nanocrystals. Chem. Mater. 15, 2854-2860 (2003).

24. Khitrova, G., Gibbs, H. M., Kira, M., Koch, S. W. \& Scherer, A. Vacuum Rabi splitting in semiconductors. Nat. Phys. 2, 81-90 (2006).

25. Chang, D. E., Vuletic, V. \& Lukin, M. D. Quantum nonlinear optics - photon by photon. Nature Photon. 8, 685-694 (2014).

26. Ridolfo, A., Di Stefano, O., Fina, N., Saija, R. \& Savasta, S. Quantum plasmonics with quantum dot-metal nanoparticle molecules: influence of the fano effect on photon statistics. Phys. Rev. Lett. 105, 263601 (2010).

27. Johnson, P. B. \& Christy, R. W. Optical constants of the noble metals. Phys. Rev. B 6, 4370-4379 (1972).

28. Koenderink, A. F. On the use of Purcell factors for plasmon antennas. Opt. Lett. 35, 4208-4210 (2010).

\section{Acknowledgements}

G.H. is the incumbent of the Hilda Pomeraniec professorial chair. We thank Dr Barak Dayan for useful comments on the manuscript.

\section{Author contributions}

K.S., O.B. and G.H. designed the research. K.S. and O.B. performed the experiments. L.C. performed electromagnetic simulations. All authors participated in writing the manuscript.

\section{Additional information}

Supplementary Information accompanies this paper at http://www.nature.com/ naturecommunications

Competing financial interests: The authors declare no competing financial interests.

Reprints and permission information is available online at http://npg.nature.com/ reprintsandpermissions/

How to cite this article: Santhosh, K. et al. Vacuum Rabi splitting in a plasmonic cavity at the single quantum emitter limit. Nat. Commun. 7:11823 doi: 10.1038/ncomms11823 (2016).

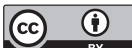

This work is licensed under a Creative Commons Attribution 4.0 International License. The images or other third party material in this article are included in the article's Creative Commons license, unless indicated otherwise in the credit line; if the material is not included under the Creative Commons license, users will need to obtain permission from the license holder to reproduce the material. To view a copy of this license, visit http://creativecommons.org/licenses/by/4.0/ 